\title{
Effects of Delay in the Snap Freezing of Colorectal Cancer Tissues on the Quality of DNA and RNA
}

\author{
Sang Hyun Hong ${ }^{1,2}$, Hyun Ah Baek ${ }^{1,2}$, Kyu Yun Jang ${ }^{2}$, Myoung Ja Chung ${ }^{1,2}$, Woo Sung Moon ${ }^{2}$, \\ Myoung Jae Kang ${ }^{2}$, Dong Geun Lee ${ }^{2}$, Ho Sung Park ${ }^{1,2}$ \\ ${ }^{1}$ Chonbuk National University Hospital National Biobank of Korea, \\ ${ }^{2}$ Department of Pathology, Research Institute of Clinical Medicine, Chonbuk National University Medical School, Jeonju, Korea
}

Purpose: The success of basic molecular research using biospecimens strongly depends on the quality of the specimen. In this study, we evaluated the effects of delayed freezing time on the stability of DNA and RNA in fresh frozen tissue from patients with colorectal cancer.

Methods: Tissues were frozen at 10, 30, 60, and 90 minutes after extirpation of colorectal cancer in 20 cases. Absorbance ratio of 260 to $280 \mathrm{~nm}\left(\mathrm{~A}_{260} / \mathrm{A}_{280}\right)$ and agarose gel electrophoresis were evaluated. In addition, the RNA integrity number (RIN) was assayed for the analysis of the RNA integrity.

Results: Regardless of delayed freezing time, all DNA and RNA samples revealed $\mathrm{A}_{260} / \mathrm{A}_{280}$ ratios of more than 1.9, and all DNA samples showed a discrete, high-molecular-weight band on agarose gel electrophoresis. The RINs were 7.53 \pm 2.04 , $6.70 \pm 1.88,6.47 \pm 2.58$, and $4.22 \pm 2.34$ at $10,30,60$, and 90 minutes, respectively. Though the concentration of RNA was not affected by delayed freezing, the RNA integrity was decreased with increasing delayed freezing time.

Conclusion: According to the RIN results, we recommend that the collection of colorectal cancer tissue should be done within 10 minutes for studies requiring RNA of high quality and within 30 minutes for usual RNA studies.

Keywords: Colorectal neoplasms; Tissue banks; DNA; RNA; Quality control

\section{INTRODUCTION}

With recent rapid development of genomics and proteomics, as well as increased demands for studies on the usefulness of biological markers, the trend of studies on diseases is changing. As a result of such changes, in the investigation of diseases, the demand for tissues samples reflecting the characteristics of diseases well and for added clinical information is on the increase more and more. Therefore, in several institutions in Korea, as well as other countries, efforts have been made to

Received: July 16, 2010 Accepted: September 6, 2010

Correspondence to: Ho Sung Park, M.D.

Department of Pathology, Chonbuk National University Medical School,

San 2-20 Geumam-dong, Deokjin-gu, Jeonju 561-180, Korea

Tel: +82-63-270-3073, Fax: +82-63-270-3135

E-mail: hspark@chonbuk.ac.kr

(C) 2010 The Korean Society of Coloproctology

This is an open-access article distributed under the terms of the Creative Commons Attribution NonCommercial License (http://creativecommons.org/licenses/by-nc/3.0) which permits unrestricted non-

commercial use, distribution, and reproduction in any medium, provided the original work is properly cited. secure sufficiently good quality tissue samples by operating tissue sample banks. Particularly, in the future, the possibility that investigators could collect tissue samples from several institutions through networks and conduct studies is high; thus, the need to provide good quality tissue samples is higher. Nevertheless, the system for collecting and storing tissue samples has not yet been standardized, and many diverse systems, depending on the conditions at the institutions, exist.

Multiple factors may determine the quality of tissue samples; however, the most important factor is the time spent to collect the tissue samples. During surgical resection of solid tumors, major blood vessels are ligated, which induces tissue ischemia and causes protein degradation and denaturation until the fixation in formalin or in liquid nitrogen. Furthermore, it has been reported that the expression of genes varies with the elapse of the ischemic time after surgical resection and that with the delay of the fixation time, the usefulness of mRNA is reduced $[1,2]$. Therefore, good quality tissue samples could be obtained by fixing tumor tissues as soon as possible after surgical resection. The interval from surgical tissue extraction 
to freezing is 30 minutes in the standard operating procedure of Tuba Frost, and the guideline of the MD Anderson Cancer Center recommends that the interval be 10 minutes $[3,4]$. Nevertheless, regarding RNA degradation, the standard for the time interval from tissue extraction to tissue freezing is not clear. In this study, through an examination of the change in the stability of DNA and RNA with changing time interval from surgical resection to freezing of colorectal cancer tissues, a time interval that must be observed to provide good quality tissue samples suitable to molecular biological studies is suggested.

\section{METHODS}

\section{Collection and storage of fresh frozen colorectal cancer tissues}

From among patients who underwent surgery for colorectal cancer at the Chonbuk National University Hospital from July 2009 to October 2009, the subjects of this study were 20 patients from whom fresh frozen tissues were collected by the Chonbuk National University Hospital (National Biobank of Korea). The subjects were patients who were not treated with chemotherapy or radiation therapy prior to surgery. Consent for the donation of specimens was obtained prior to surgery, and tissue samples were collected only from patients who signed a consent form for the donation of specimens. Tissues extracted in operating rooms were placed in an icebox maintained at $4^{\circ} \mathrm{C}$ by adding ice in advance and were transported to the gross examination room. After gross examination, tumor tissues were collected in a range that would not affect di-

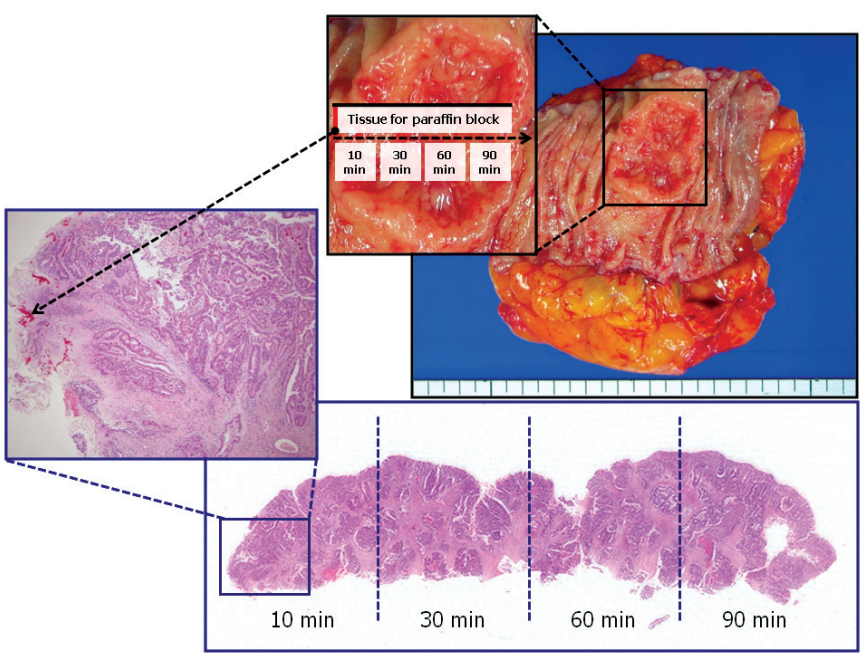

Fig. 1. Sampling protocol and marking for tissue orientation. From left to right, tissues for snap-freezing were taken at 10, 30, 60, and 90 minutes after extirpation of colorectal cancer. A mirror-imaged tissue for the paraffin-embedded block was taken, and it was painted with red ink on the left end and with black ink at the opposite side of the cut surface. agnosis. The tumors were sectioned in the midline, and while starting from the left side and advancing to the right side, 4 consecutive tissues $0.5 \times 0.5 \times 0.5 \mathrm{~cm}^{3}$ in size were collected (Fig. 1). The tissues collected 10 minutes, 30 minutes, 60 minutes, or 90 minutes after extraction were rapidly frozen with liquid nitrogen by adding it to isopentane chilled in advance and were subsequently stored in a liquid-nitrogen tank .

\section{Preparation of the mirror image slide of fresh frozen tissues and evaluation}

To examine the adequacy of fresh frozen tissues for use in experiments, we prepared hematoxylin-eosin slides of the mirror image area of fresh frozen tissues, and we evaluated the adequacy of the frozen samples. For the preparation of precise mirror image slides, sections were obtained from tissues sharing the cross section of the area harvested by the fresh frozen tissues. The left end of the tissue surface opposite to tissues frozen after 10 minutes was marked with red ink and paired with the collected tissues, and the side without contact to tissues harvested at each time point was marked with black ink, and during paraffin embedding, the orientation was maintained to allow the side without marking with black ink to be microsected (Fig. 1). Paraffin blocks of mirror image tissue sections were prepared, and their adequacy was evaluated by hematoxylin-eosin staining. Concerning the categories for the evaluation of adequacy and the standard, by referring to the result of the standardization of the Korean human sample management guideline, the quantity of included tumors (less than $50 \%$ is not adequate), contamination of tissues (tissues without contamination of other tissues are excellent), the quantity of necrosis (more than 50\% area of the entire slide is not suitable), the quantity of extracellular mucin (tissue with a quantity of mucin more than $50 \%$ of the entire slide is not suitable), and inflammatory reaction in the interstitial space were evaluated [5].

\section{Evaluation of the stability of DNA and RNA of fresh frozen tissues}

DNA and RNA were extracted from fresh frozen tissues, and the stability of fresh frozen tissues was evaluated by using a quantitative analysis and an analysis of integrity. Fresh frozen tissues were divided and added to 1.5-mL microcentrifuge tubes, and DNA was extracted by using a QIAamp DNA mini kit (Qiagen, Hilden, Germany) according to the method suggested by the manufacturer. RNA was extracted using the RNeasy protect mini kit (Qiagen) according to the method suggested by the manufacturer. For the quantitative analysis of the extracted DNA and RNA, the ratio of $\mathrm{A}_{260} / \mathrm{A}_{280}$ was measured through the use of the NanoDrop (Thermo Fisher Scientific Inc, Waltham, MA, USA). Cases with a ratio of $\mathrm{A}_{260} / \mathrm{A}_{280}$ higher than 1.8 were evaluated as excellent, cases with a ratio between 1.6 and 1.8 were adequate, and cases with a ratio of less than 
1.6 were inadequate. The integrity of DNA was evaluated by using agarose gel electrophoresis, and if distinct high-molecular-weight bands were observed in electrophoresis, it was evaluated to be adequate. The integrity of RNA was evaluated by measuring the $28 \mathrm{~s}$ and the 18 s ribosomal RNA (rRNA) bands shown on agarose gel electrophoresis by using the Multi Gauge V3.0 program (FujiFilm, Tokyo, Japan) and by measuring the RNA integrity number (RIN) through the use of the Agilent 2100 Bioanalyzer (Agilent Technologies, Santa Clara, CA, USA). On electrophoresis, the $28 \mathrm{~s}$ and the $18 \mathrm{~s}$ rRNA bands were examined, and the ratio of the 28 s to the $18 \mathrm{~s}$ rRNA band was recorded as a mean \pm standard deviation. A RIN higher than 7 was evaluated to be 'excellent', a RIN between 4 and 7 was 'good,' and a RIN lower than 4 was 'bad' [5-9]. The Agilent 2100 Bioanalyzer is an automated bioanalyzer that applies microfluidics techniques, and it consists of microdifluidic chips, gel-filled channels, equipment for classifying voltage-induced molecular weights in the gel-filled channel, and a laser-induced fluorescence detection system. When a small amount of RNA sample is classified according to molecular weight within microfibrillar chips and detected by laser-induced fluorescence detection, the result is presented as electropherograms.
The RIN is calculated by using the "RIN software algorithm" and is shown as a number from 1 to 10. Complete RNA is shown as RIN 10. As RNA is degraded, the RIN becomes lower, and completely degraded RNA is shown as RIN 1 [8]. The RIN is presented as numbers; thus, it has advantages in that it provides objective evaluation data on RNA integrity and suggests the range of the use of RNA; nonetheless, it has the shortcoming of requiring expensive equipment.

\section{Statistical analysis}

Statistical analysis was performed by using SPSS ver.15.0 (SPSS Inc., Chicago, IL, USA). The differences in the $\mathrm{A}_{260} / \mathrm{A}_{280}$ ratio of DNA, the $A_{260} / A_{280}$ ratio of RNA, the ratio of the $28 \mathrm{~s}$ to the 18 s rRNA band, and the RIN value between the groups was validated by using the one-way ANOVA test, and a P-value less than 0.05 was considered to be statistically significant.

\section{RESULTS}

\section{Evaluation of the adequacy of the collection area of fresh frozen tissues}

The results of the evaluation of the adequacy of the area col-

Table 1. Histopathologic evaluation of the sampling adequacy for fresh frozen tissue

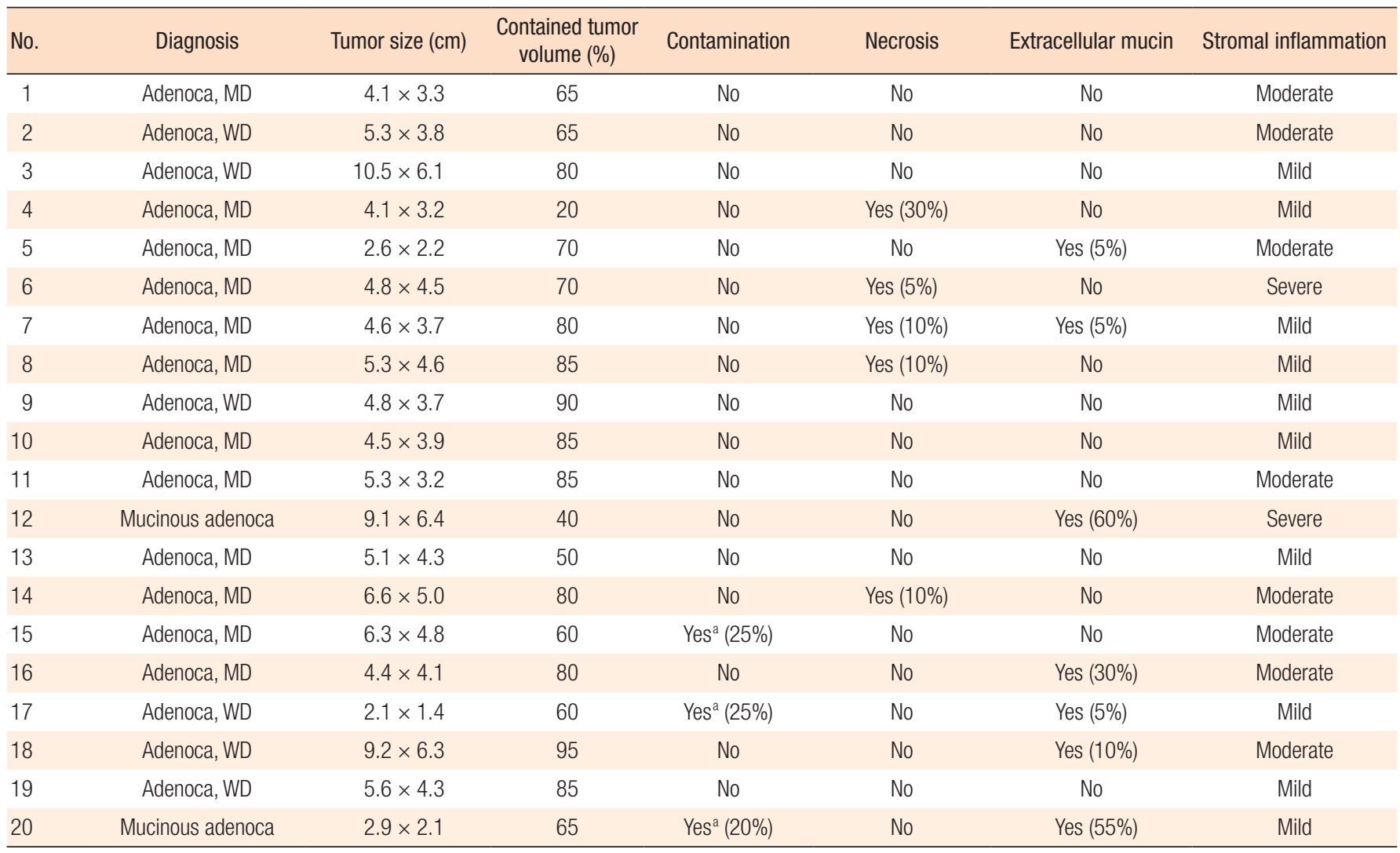

Adenoca, adenocarcinoma; MD, moderately differentiated; WD, well differentiated.

aPresence of normal mucosal tissue. 
lected by the fresh frozen tissues are shown in Table 1. Two samples (10\%) contained less than 50\% tumor cells, 3 samples (15\%) showed tissue contamination, no samples had more than $50 \%$ necrosis, 2 samples (10\%) showed 50\% extracellular mucin, and 2 samples (10\%) showed a severe interstitial inflammatory reaction. The results for the adequacy of the area collected by the fresh frozen tissues show that in a total 20 cases of colorectal cancer samples, 15 cases (75\%) were determined to be adequate. Even for samples for which the collection area was not adequate, this was thought not to affect the stability of the fresh frozen tissues; thus, the stabilities of DNA and RNA of all 20 cases were evaluated.

\section{Evaluation of the stability of DNA for fresh frozen tissues}

DNA was extracted from fresh frozen tissues, and the $A_{260} / A_{280}$ ratio was measured. The ratio for tissues frozen 10 minutes after extraction was $1.95 \pm 0.04$, that for tissues frozen $30 \mathrm{~min}$ utes after extraction was $1.92 \pm 0.06$, that for tissues frozen after 60 minutes was $1.93 \pm 0.05$, and that for tissues frozen $90 \mathrm{~min}$ utes after extraction was $1.92 \pm 0.05$ (Fig. 2A); no statistical differences were detected $(\mathrm{P}=0.258)$. Regardless of the time interval from extraction to freezing, the $\mathrm{A}_{260} / \mathrm{A}_{280}$ ratio of all tissues was higher than 1.8 and this ratio was determined to be 'excellent.' In agarose gel electrophoresis of DNA, all tissues showed distinct high-molecular-weight bands; thus, regardless of the elapsed time from extraction to freezing, the integrity of all tissues was evaluated to be 'adequate' (Fig. 2B).

\section{Evaluation of the stability of RNA for fresh frozen tissues}

RNA was extracted from fresh frozen tissues, and the $A_{260} / A_{280}$
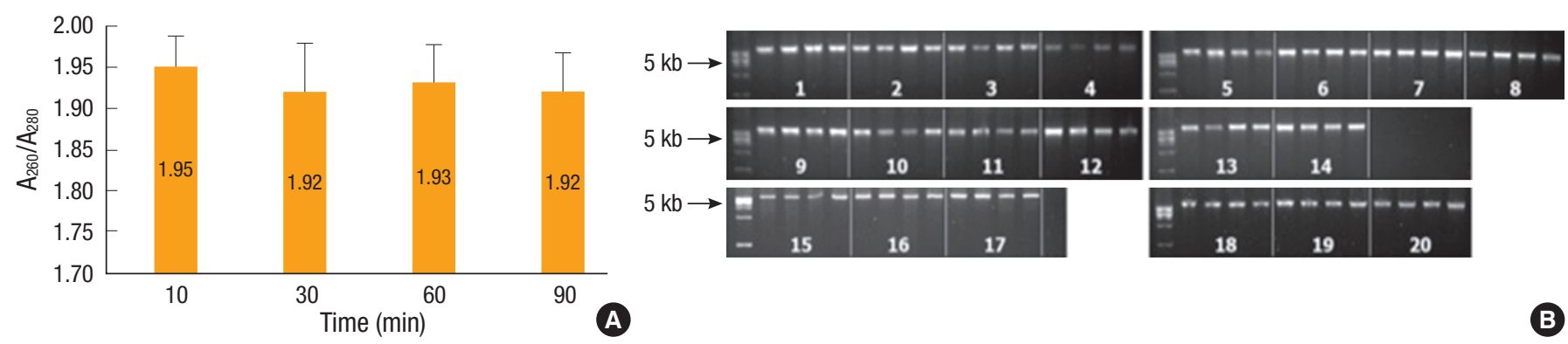

Fig. 2. Purity and integrity of DNA in 20 cases of colorectal carcinoma tissues. (A) The means of the $\mathrm{A}_{260} / \mathrm{A}_{280}$ ratio are over 1.8 regardless of delayed freezing time, and all DNA samples are judged excellent. (B) In agarose gel electrophoresis, all cases of DNA samples reveal a discrete high molecular weight band. In each case, the lanes are in order of delayed time 10, 30, 60, and 90 minutes, respectively.

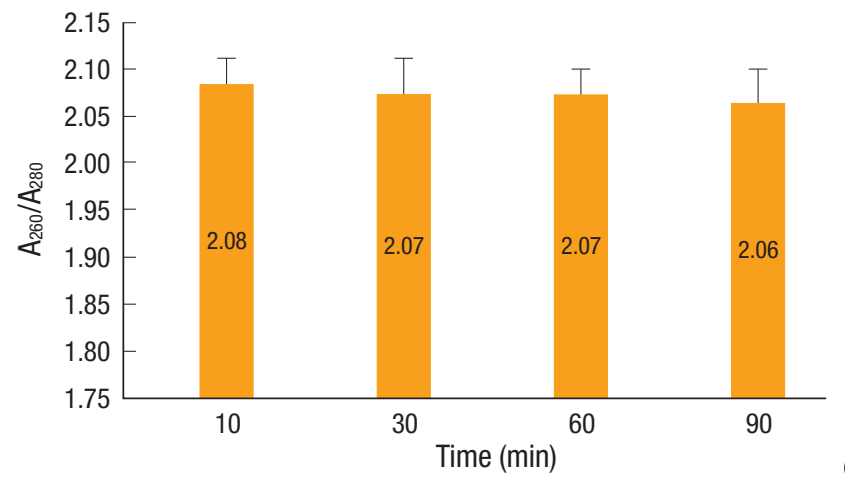

A
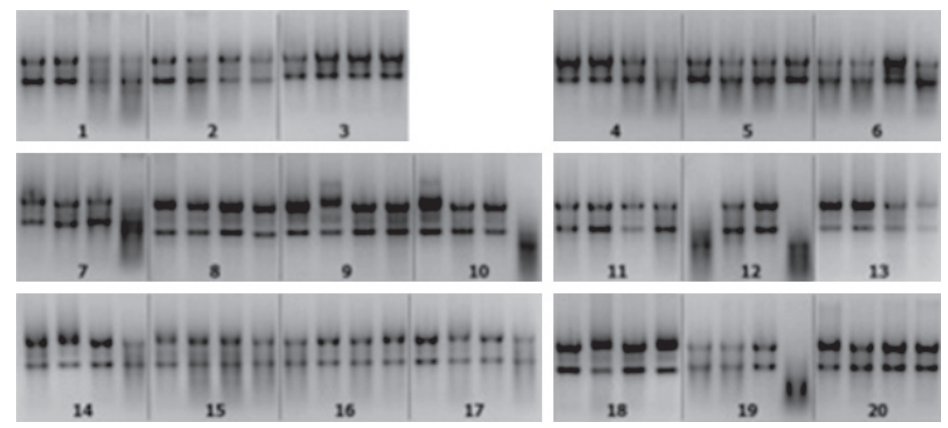

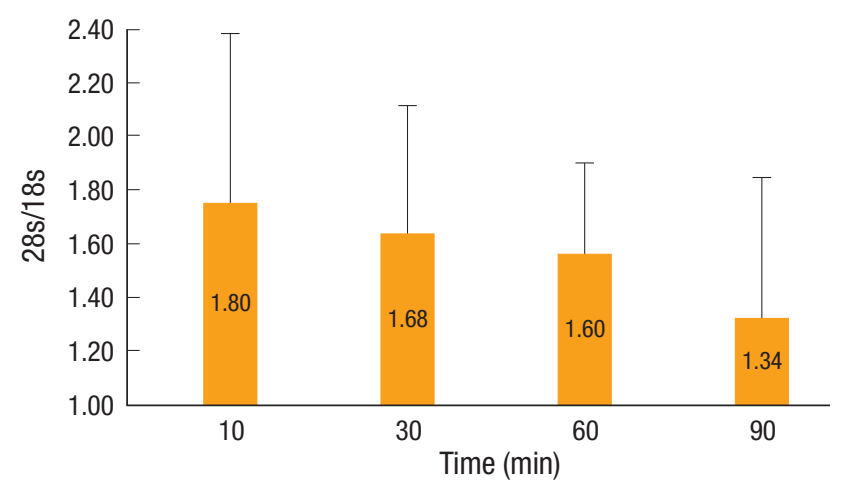

C
Fig. 3. Purity and integrity of RNA in 20 cases of colorectal carcinoma tissues. (A) The means of the $\mathrm{A}_{260} / \mathrm{A}_{280}$ ratio are over 1.8 regardless of delayed freezing time, and all RNA samples are judged excellent. (B) In agarose gel electrophoresis, $28 \mathrm{~s}$ and $18 \mathrm{~s}$ ribosomal RNA bands are shown. In each case, the lanes are in order of delayed time 10, 30, 60, and 90 minutes, respectively. (C) The means of the $28 \mathrm{~s} / 18 \mathrm{~s}$ ratio gradually decrease with increasing delayed freezing time.

. 

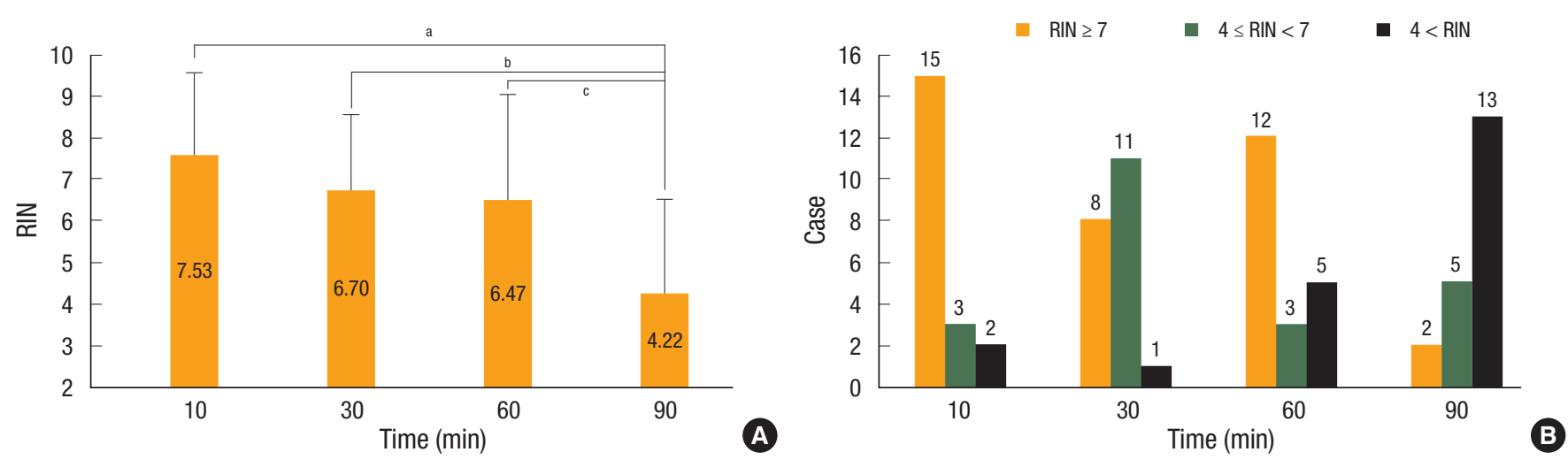

Fig. 4. (A) The means of the RNA integrity number (RIN) gradually decrease with increasing delayed freezing time: ${ }^{\mathrm{a}} \mathrm{P}=0.000 ;{ }^{\mathrm{b}} \mathrm{P}=0.004 ;{ }^{\mathrm{c}} \mathrm{P}$ $=0.011$. (B) Case distribution of RIN according to the delayed time.

ratio was measured. The ratio of tissues frozen 10 minutes after extraction was $2.08 \pm 0.03$, that of tissues frozen 30 minutes after extraction was $2.07 \pm 0.04$, that of tissues frozen $60 \mathrm{~min}$ utes after extraction was $2.07 \pm 0.03$, and that of tissues frozen 90 minutes after extraction was $2.06 \pm 0.04$ (Fig. 3A); no statistical differences were detected $(\mathrm{P}=0.182)$. Regardless of the time from extraction to freezing, the $\mathrm{A}_{260} / \mathrm{A}_{280}$ ratio of all tissues was higher than 1.8 , and this ratio was determined to be 'excellent.' In RNA agarose gel electrophoresis, in tissues frozen 10 minutes after extraction, the $28 \mathrm{~s}$ and the $18 \mathrm{~s}$ rRNA bands were observed in 19 cases, and the bands could not be distinguished in 1 case. In tissues frozen 30 minutes and 60 minutes after extraction, in all 20 cases, the $28 \mathrm{~s}$ and the $18 \mathrm{~s}$ rRNA bands were observed. In tissues frozen 90 minutes after extraction, the $28 \mathrm{~s}$ and the $18 \mathrm{~s}$ rRNA band were observed in 16 cases, and the bands could not be distinguished in 4 cases (Fig. 3B). The ratio of the 28s to the $18 \mathrm{~s}$ rRNA band of samples frozen $10 \mathrm{~min}$ utes after extraction was $1.80 \pm 0.68$, that of tissues frozen 30 after extraction was $1.68 \pm 0.52$, that of tissues frozen $60 \mathrm{~min}$ utes after extraction was $1.60 \pm 0.37$, and that of tissues frozen 90 minutes after extraction was $1.34 \pm 0.57$ (Fig. 3C); no statistical differences were observed. Nonetheless, in tissues frozen 10 minutes after extraction, the ratio of the $28 \mathrm{~s}$ to the $18 \mathrm{~s}$ rRNA band showed a tendency to be higher in comparison with tissues frozen 90 minutes after extraction $(\mathrm{P}=0.058)$.

The RIN of tissues frozen 10 minutes after extraction was $7.53 \pm 2.04$, that of tissues frozen 30 minutes after extraction was $6.70 \pm 1.88$, that of tissues frozen 60 minutes after extraction was $6.47 \pm 2.58$, and that of tissues frozen 90 minutes after extraction was $4.22 \pm 2.34$ (Fig. $4 \mathrm{~A}$ ). The RIN according to the interval from extraction to freezing was statistically different. The RIN of tissues frozen 10 minutes after extraction was higher than that of tissues frozen 90 minutes after extraction $(\mathrm{P}=0.000)$, the RIN of tissues frozen 30 minutes after extraction was also higher than that of tissues frozen 90 minutes after extraction ( $P=0.004)$, and the RIN of tissues frozen 60 minutes after extraction was also higher than that of tissues frozen
90 minutes after extraction $(\mathrm{P}=0.011)$. On the other hand, for each time interval, 10, 30, 60, and 90 minutes, the numbers of 'excellent' specimen were 15 cases, 8 cases, 12 cases, and 2 cases, respectively. The numbers of 'good' specimens was $3,11,3$, and 5 , respectively. The numbers of 'poor' specimens were 2, 1, 5, and 13, respectively (Fig. 4B).

\section{DISCUSSION}

In our study, when fresh frozen tissues were collected from colorectal cancer, the adequacy of the harvested area was evaluated, and according to the interval from surgical extraction to freezing, the change in the stability of DNA and RNA was evaluated. Since fresh frozen tissues are used primarily for the extraction of DNA, RNA and protein, an evaluation of the adequacy of frozen tissues, such as whether the tissues harvested from tumors reflect the characteristics of the harvested area, whether is the tissues are contaminated, etc., must be performed prior to the use of the tissue samples.

The mirror image tissues adjacent to the fresh frozen tissues were embedded in paraffin, prepared as slides, and read under light microscope and the adequacy of the harvested area was evaluated. Among 20 cases, 15 cases were adequate, and 5 cases were inadequate. It is a level comparable to other reports. Sandusky et al. [10] reported that among 480 specimens collected from various cancer tissues, samples containing less than $65 \%$ tumor components were $25 \%$, and particularly, in colorectal cancer tissues, specimens containing less than $65 \%$ tumor components were $32.2 \%$. In our study, two specimens had a quantity of tumor cells less than $50 \%$ and were, thus, determined to be inadequate. Among them, one contained substantially more interstitial components than tumor cells and it was an adenocarcinoma associated with substantial necrosis (case 4). The other case was a mucinous carcinoma (case 12). A mucinous carcinoma is defined as a tumor that has more than a $50 \%$ mucinous component; thus, in regard to the case, whether it is reasonable to determine the case as inadequate because the 
quantity of tumor cells is small must be addressed.

Three samples were determined to be inadequate because of the contamination of tissues, and all of them contained more than $20 \%$ of normal mucosal tissues. Among them, the long diameters of the tumors of 2 cases was smaller than $3 \mathrm{~cm}$; thus, in comparison with other cases, the size of tumors was small (cases 17 and 20), and due to this, normal mucosal tissues were contained. Therefore, for the collection of tumor tissues, more comprehensive attention and skilled gross examination are required. It is thought that a flexible guideline to regulate the number of tissues to be harvested, depending on the size of tumors, is required. In the two cases, the quantity of extracellular mucin was more than $50 \%$; thus, the samples were determined to be inadequate, but both cases were mucinous carcinomas (cases 12 and 20). Even if tissues are determined to be inadequate due to a small amount of tumors, contamination with other tissues, necrosis, or large amount of extracellular mucin, the tissues could be used for investigations by the application of the laser capture microdissection method, which allows tumor cells to be harvested selectively; thus, a more precise and detailed description is required to read the mirror image slides.

When DNA and RNA are extracted from fresh frozen tissues, a purity test, as well as integration test, is performed. Nucleic acid has the characteristic of absorbing ultraviolet light in specific patterns. When $260-\mathrm{nm}$ wavelength ultraviolet light is irradiated, as the concentration of nucleic acid in samples becomes higher, the ultraviolet absorbance also becomes higher. In nucleic acid extraction samples, contamination with protein, organic components and other molecules is frequent, and proteins absorb the 280-nm wavelength. Therefore, the purity test applies the ratio of the absorbance at a $260-\mathrm{nm}$ wavelength to that at a $280-\mathrm{nm}$ wavelength. The lower the $\mathrm{A}_{260} / \mathrm{A}_{280}$ ratio is, the higher the protein contamination. In our study, regardless of the elapsed time from tissue extraction to freezing, the $\mathrm{A}_{260} /$ $\mathrm{A}_{280}$ ratio of all samples was higher than 1.8 , and the samples were determined to be 'excellent.'

The methods evaluating the integrity of DNA are electrophoresis, Southern analysis, gene-specific polymerase chain reaction (PCR), multiple gene-specific PCR, randomly amplified polymorphic DNA (RAPD)-PCR, etc. [11]. Among them, electrophoresis is a method examining DNA fragmentation. Since it is inexpensive in comparison with other methods, it has been used widely [11]. In our study, the electrophoresis method was applied to evaluate DNA integrity, and regardless of the elapsed time from tissue extraction to freezing, all tissues were determined to be 'adequate.' Therefore, the DNA of colorectal cancer tissues was found to be stably maintained up to $90 \mathrm{~min}$ utes after extraction.

There are two methods for evaluating the integrity of RNA. One is the method assessing the ratio of the $28 \mathrm{~s}$ to the $18 \mathrm{~s}$ RNA band after agarose gel electrophoresis, and the other is the method measuring the RIN by using a bioanalyzer. rRNA accounts for more than $80 \%$ of the entire RNA, so it can be assessed readily. On the other hand, mRNA is only $1-3 \%$ of the entire RNA, so even if sensitive methods are applied, mRNA detection is not easy. Based on many studies inferring that the quality and the quantity of rRNA reflect the quality and the quantity of mRNA, the quality of mRNA has been evaluated by using electrophoresis of the entire RNA. Generally, when the ratio of the $28 \mathrm{~s}$ to the $18 \mathrm{~s}$ rRNA band is 2.0 , RNA is considered to be intact. RNA is degraded readily by unskilled treatments of specimens and by destruction due to ischemia, apoptosis, necrosis, etc. The $28 \mathrm{~s}$ rRNA is bigger than the $18 \mathrm{~s}$ rRNA, and the structure of 28s rRNA is more unstable; thus, $28 \mathrm{~s}$ rRNA is degraded more than $18 \mathrm{~s}$ rRNA. Therefore, it is very difficult to obtain a ratio of the $28 \mathrm{~s}$ to the $18 \mathrm{~s}$ rRNA band of 2.0, and in the study reported by Sandusky et al. [10] in 75\% of 1,550 specimens, the ratio of the $28 \mathrm{~s}$ to the $18 \mathrm{~s}$ band was found to be in the range of 1.5-1.8. In addition, Dumur et al. [12] concluded that for the analysis of a gene array, samples with a ratio of the $28 \mathrm{~s}$ to the $18 \mathrm{~s}$ rRNA band of higher than 1.4 were adequate, and some studies showed that the ratio of the $28 \mathrm{~s}$ to the $18 \mathrm{~s}$ rRNA band was not adequate to evaluate the quality and the usefulness of RNA $[1,13,14]$. In our study, the $\mathrm{A}_{260} / \mathrm{A}_{280}$ ratios for various elapse times from colorectal cancer tissue extraction to freezing were not different, which shows that the purity level of the extracted RNA was high, without protein contamination. In addition, the ratio of the 28 s to the 18s rRNA band of samples frozen 10 minutes after the extraction of colorectal cancer tissues was highest. Since $28 \mathrm{~s}$ rRNA is degraded more than 18s rRNA with time, the ratio of the $28 \mathrm{~s}$ to the $18 \mathrm{~s}$ rRNA band is decreased, and by freezing the samples within a short time after extraction, good quality RNA could be obtained. On the other hand, in agarose gel electrophoresis, among samples frozen 10 minutes after extraction, the $28 \mathrm{~s}$ and the 18s rRNA bands could not be distinguished in 1 case (case 12), a mucinous carcinoma with severe infiltration of inflammatory cells. Therefore, inflammation, mucin, etc. are thought to have effects on the stability of RNA. Nonetheless, in other cases showing inflammation, mucin, etc., the $28 \mathrm{~s}$ and the $18 \mathrm{~s}$ rRNA bands could be distinguished; thus, the possibility of carelessness during the specimen treatment and RNA extraction is higher than the effect of inflammation and mucin.

The intensity of the rRNA band is affected by the condition of electrophoresis, the amount of loaded RNA, and the saturation level of ethidium bromide; thus, subjective results are obtained by using a gross evaluation of the ratio of the $28 \mathrm{~s}$ to the $18 \mathrm{~s}$ band on the agarose gel. Hence, recently, studies evaluating RNA integrity by using a bioanalyzer to measuring the RIN have been on the rise. Fleige et al. [15] measured the RINs of various bovine organs, and they reported that in comparison with other organs, lower values were obtained from gastrointestinal tissues, which was due to their containing a large 
quantity of connective tissues; thus, a large amount of RNA was degraded during the tissue collection and extraction processes. In addition, depending on the organ, the RIN shows diverse results from 4.56 to 9.62 , depending on whether tissues are hard (whether tissues contain connective tissues or adipose tissues), the difference of RNase enzyme activity, etc [15]. Human tissue with a RIN higher than 7 ('excellent') could be used for studies requiring high-quality RNA, such as gene array assays, and that with a RIN between 4 - 7 ('good') could be used for studies applying quantitative RT-PCR [9]. In our study, the RIN was highest in samples frozen 10 minutes after colorectal tissue extraction, and with increasing elapsed time, the RIN was decreased. Thus, if samples were frozen within a short time after tissue extraction, good quality RNA could be obtained. Particularly, at the four time points, 10, 30, 60, and 90 minutes, the numbers of specimens for which the RIN was determined to be 'excellent' were 15 cases, 8 cases, 12 cases, and 2 cases, respectively. Hence, for gene array assay, it is better to freeze samples within 10 minutes after extraction. The numbers of specimens determined to be 'good' or 'excellent' were 18 cases, 19 cases, 15 cases, and 7 cases, respectively; thus, for studies applying quantitative RT-PCR, it is better to freeze samples within 30 minutes after extract. For specimens frozen 30 minutes - 60 minutes after extraction, it would be better to use them after confirmation by RNA quantification and integrity analysis. Our results on the adequacy of times from extraction to freezing were comparable to those of several other studies. Spruessel et al. [16] reported that for freezing 5-8 minutes after colorectal resection, early changes in the expression of genes and proteins were observed, and after 30 minutes, 20\% of detectable genes and proteins showed important changes in expression. Huang et al. [1] reported that the stable expression of genes was shown within 20 minutes of ischemic time, and that after 40 minutes, important changes in the expression of genes were shown.

In colorectal tissues, the change in the stability of DNA and RNA with the elapsed time from surgical extraction to freezing was examined. DNA was found to be stable for up to $90 \mathrm{~min}$ utes after extraction. The stability of RNA, on the other hand, decreased with increasing time elapsed after extraction. Based on the results, there were differences in the suitable elapsed time between extraction and freezing according to RNA research method, and for gene array assay, freezing within 10 minutes is thought to be required, and for studies applying quantitative RT-PCR, freezing within 30 minutes is thought to be required. Nevertheless, the stability of RNA becomes lower as the time until freezing is delayed, so tissues should be collected and treated within as short a time as possible, and high quality RNA can be obtained by paying more attentions to the RNA extraction process.

\section{CONFLICT OF INTEREST}

No potential conflict of interest relevant to this article was reported.

\section{ACKNOWLEDGMENTS}

Fresh frozen tissue samples were provided by the Chonbuk National University Hospital, a member of the National Biobank of Korea, which is supported by the Ministry of Health, Welfare and Family Affairs. This work was supported by the fund of "The establishment of quality controls for solid tissue of the National Biobank of Korea" and by the Ministry of Science \& Technology (MoST)/Korea Science \& Engineering Foundation (KOSEF) through the Diabetes Research Center at Chonbuk National University (R13-2008-005-0000-0).

\section{REFERENCES}

1. Huang J, Qi R, Quackenbush J, Dauway E, Lazaridis E, Yeatman T. Effects of ischemia on gene expression. J Surg Res 2001;99:222-7.

2. Almeida A, Paul Thiery J, Magdelenat H, Radvanyi F. Gene expression analysis by real-time reverse transcription polymerase chain reaction: influence of tissue handling. Anal Biochem 2004; 328:101-8.

3. Mager SR, Oomen MH, Morente MM, Ratcliffe C, Knox K, Kerr DJ, et al. Standard operating procedure for the collection of fresh frozen tissue samples. Eur J Cancer 2007;43:828-34.

4. Mishra A, Pandey A, Shaw R. Initiating tumor banking for translational research: MD Anderson and Liverpool experience. Indian J Cancer 2007;44:17-24.

5. Choi C. Development of standard operation manual for national biobank of Korea. Seoul: Korea Centers for Disease Control and Prevention; 2008. Report No. 2008-E00353-00.

6. Elliott P, Peakman TC; UK Biobank. The UK Biobank sample handling and storage protocol for the collection, processing and archiving of human blood and urine. Int J Epidemiol 2008;37: 234-44.

7. Glasel JA. Validity of nucleic acid purities monitored by $260 \mathrm{~nm} /$ $280 \mathrm{~nm}$ absorbance ratios. Biotechniques 1995;18:62-3.

8. Schroeder A, Mueller O, Stocker S, Salowsky R, Leiber M, Gassmann $\mathrm{M}$, et al. The RIN: an RNA integrity number for assigning integrity values to RNA measurements. BMC Mol Biol 2006;7:3.

9. Asterand C. RNA quality assurance using RIN [Internet]. Detroit, MI: Asterand plc; 2006 [cited 2010 Oct 3] Available from: http://www.asterand.com/asterand/human_tissues/Asterand_ RIN.pdf.

10. Sandusky GE, Teheny KH, Esterman M, Hanson J, Williams SD. Quality control of human tissues--experience from the Indiana University Cancer Center-Lilly Research Labs human tissue bank. Cell Tissue Bank 2007;8:287-95.

11. Siwoski A, Ishkanian A, Garnis C, Zhang L, Rosin M, Lam WL. 
An efficient method for the assessment of DNA quality of archival microdissected specimens. Mod Pathol 2002;15:889-92.

12. Dumur CI, Nasim S, Best AM, Archer KJ, Ladd AC, Mas VR, et al. Evaluation of quality-control criteria for microarray gene expression analysis. Clin Chem 2004;50:1994-2002.

13. Imbeaud S, Graudens E, Boulanger V, Barlet X, Zaborski P, Eveno E, et al. Towards standardization of RNA quality assessment using user-independent classifiers of microcapillary electrophoresis traces. Nucleic Acids Res 2005;33:e56.
14. Alhasan SA, Shen X, Azrak G, Eliason JF. Analysis of RNA quality and yields of frozen samples in a large research repository. Proc Am Assoc Cancer Res 2006;47:Abstract \#841.

15. Fleige S, Pfaffl MW. RNA integrity and the effect on the real-time qRT-PCR performance. Mol Aspects Med 2006;27:126-39.

16. Spruessel A, Steimann G, Jung M, Lee SA, Carr T, Fentz AK, et al. Tissue ischemia time affects gene and protein expression patterns within minutes following surgical tumor excision. Biotechniques 2004;36:1030-7. 